\title{
A numerical model of elasticity for cardiovascular system that includes 2/3D displacements and deformations
}

\author{
Ayoub Azzayani* \\ Finance, Entrepreneurship and Development Laboratory, Faculty of Legal, Economic and Social Sciences of SALE, \\ Mohammed V University, Rabat, Morocco
}

Received: 13 June 2020 / Accepted: 30 July 2020

\begin{abstract}
In this work, we deal an elasticity model in 2D and 3D dimension for deformation under constraint by taking into account the direction of the deformation displacement. This work is a result of an article [A. Azzayani et al., Br. J. Math. Comput. Sci. (2016)] in which we use the same mathematical model by fixing the fiber orientation. Then, if we considered the case of eignvalue and eigenvector, and which is the case of this work, we can be able to control the deformation of the heart in the image processing. This mathematical model can be used to describe the heart deformation taking into account the orientation of the fibers for estimating global and regional parameters of the left ventricular function. In first, we start by presenting the proposed mathematical model on a domain $\Omega \subset \mathbb{R}^{n}(n=2$ or 3$)$, and we give the existence and uniqueness of solution to the mathematical model is given (in both $2 \mathrm{D}$ and $3 \mathrm{D}$ dimension). Secondly, we give numerical simulations with FreeFem software, simulations results and comments are given in the end. In the end, we will discuss about the image treatment with this model and its feasibility to help doctors in the diagnosis of heart disease.
\end{abstract}

Keywords: Elasticity / eigenvalues / eigenvectors / orientation / deformation / simulation

\section{Introduction}

Cardiovascular disease is a major disease and the leading cause of death in industrialized countries. In this perspective, the evolution of the treatment of this disease has grown strongly in recent decades, particularly in cardiac image processing, which allows doctors to give a more reliable and useful diagnosis to treat this type of disease [1].

Understanding and modeling of the cardiac system require a multidisciplinary approach integrating knowledge of the physics and specificities of data acquisition systems, the physiology and physio-pathology of the heart and the cardiovascular system, signal processing and image processing.

When we say signal processing and image processing, we talk about restoration and segmentation of cardiac image.

This image processing remains a time-consuming task, and of course subject to considerable variability within and between operators. Research in the field of medical image processing aimed at making the process of analyzing

\footnotetext{
* e-mail: ayoubsaha@gmail.com
}

specific images faster and more automatic to reduce subjectivity and time required for implementation of the algorithms [2].

Then, the aim of this work is, in first to continues which is been treated in article [3], and second to make this study more close to reality by treating the case of eigenvalues and eigenvectors. To better explain, we know that in reality, we know that when taking a cardiac image, the heart deforms in several directions, and therefore the difficulty that appears here and to control this direction to better have a correct image (not blurred) and define well if there is an anomaly that appears.

For this, we represent a section of the left ventricle of the heart by $\Omega$, an open bounded of $\mathbb{R}^{n}(n=2$ or 3 ), whose boundary is denoted by $\Gamma$.

We denote by $u$ the vector that describes the displacement of a material point $\mathrm{x}$ in $\Omega$, and $\mathrm{K}$ a part of $\Omega$, where in the constraint is verified.

The first section is devoted to give all the theoretical analyzes of the proposed model of elasticity with constraints [4-6].

In the second section, and before the conclusion, the proposed model is validated by numerical simulations $[7,8]$, which has been performed with Free Fem software [9]. The interpretation of those simulations is given. 


\section{The elasticity model with stress fiber orientation}

\subsection{The mathematical model}

In this section, we focus on the case where the displacement $\mathrm{u}$ is described by a model of elasticity in both cases $2 \mathrm{D}$ and $3 \mathrm{D}$ dimension, taking into account the orientation of the fibers in the heart deformation. Let $n \in 2,3$ and $\Omega$ an open bounded domain in $\mathbb{R}^{n}$. We define the strain tensor:

$$
\varepsilon(u)=\left(\begin{array}{cccc}
\varepsilon_{11}(u) & \varepsilon_{12}(u) & \ldots & \varepsilon_{1 n}(u) \\
\varepsilon_{21}(u) & \varepsilon_{22}(u) & \ldots & \varepsilon_{2 n}(u) \\
: & : & \ldots & : \\
: & : & \ldots & : \\
\varepsilon_{n 1}(u) & \varepsilon_{n 2}(u) & \ldots & \varepsilon_{n n}(u)
\end{array}\right)
$$

where $\varepsilon_{i j}(u)=\frac{1}{2}\left(\frac{\partial u_{i}}{\partial x_{j}}+\frac{\partial u_{j}}{\partial x_{i}}\right)$ and the stress tensor

$$
\sigma(u)=\left(\begin{array}{cccc}
\sigma_{11}(u) & \sigma_{12}(u) & \ldots & \sigma_{1 n}(u) \\
\sigma_{21}(u) & \sigma_{22}(u) & \ldots & \sigma_{2 n}(u) \\
: & : & \ldots & : \\
: & : & \ldots & : \\
\sigma_{n 1}(u) & \sigma_{n 2}(u) & \ldots & \sigma_{n n}(u)
\end{array}\right)
$$

where $\sigma_{i j}(u)=\beta\left(\sum_{k=1}^{n} \varepsilon_{k k}(u)\right) \delta_{i j}+2 \mu \varepsilon_{i j}(u)$.

The parameters $\beta \geq 0$ and $\mu>0$ denote the Lame constants. The proposed mathematical model is defined as follow:

$$
\begin{cases}-\operatorname{div} \sigma(u)=f & \text { in } \Omega, \\ \varepsilon(u) w=\theta w & \text { in } K \subset \Omega, \\ u=0 & \text { in } \Gamma,\end{cases}
$$

$K$ is the part of $\Omega$, where in the constraint is verified and $\mathrm{w}$ is the associate eigenvector of eigenvalue $\theta$ and represent the orienting displacement. follows:

The weak formulation of the problem (1) is written as

$$
\begin{cases}\int_{\Omega}[(\beta[\operatorname{div}(u) \cdot \operatorname{div}(v)+2 \mu \varepsilon(u): \varepsilon(u)] d x & \forall v \in\left(H_{0}^{1}(\Omega)\right)^{2} \\ \quad+\int_{K} \lambda(\varepsilon(v) \cdot w) \lambda d x=\int_{\Omega} f \cdot v d x & \\ \int_{K}(\varepsilon(v) \cdot w) q d x=\int_{K}(\theta \cdot w) q d x & \forall q \in\left(L^{2}(K)\right)^{2}\end{cases}
$$

Now, we proceed to solve the problem (2) by the Lagrangian optimization method as defined in [10,11], and we have the following problem:

$$
\min _{u \in\left(H_{0}^{1}(\Omega)\right)^{2}} \max _{\lambda \in\left(L^{2}(K)\right)^{2}} L(v, \eta)
$$

with:

$$
\begin{aligned}
L(u, \lambda)= & \int_{\Omega}\left[\left(\beta(\operatorname{div}(u))^{2}+2 \mu(\varepsilon(u))^{2}\right] d x-\int_{\Omega} f \cdot u d x\right. \\
& +\int_{K}(\varepsilon(u) \cdot w) \lambda d x+\int_{K}(\varepsilon(u) \cdot w) q d x-\int_{K}(\theta \cdot w) q d x
\end{aligned}
$$

$\lambda$ is the Lagrange multiplier.

Let define the functional $F$ as, for all $w \in L^{2}(K)^{n}$, we have:

$$
\begin{aligned}
F(w)= & \int_{\Omega}\left[\left(\beta(\operatorname{div}(u))^{2}+2 \mu(\varepsilon(u))^{2}\right] d x-\int_{\Omega} f \cdot u d x\right. \\
& +\int_{K}(\varepsilon(u) \cdot w) \lambda d x+\int_{K}(\varepsilon(u) \cdot w) q d x-\int_{K}(\theta \cdot w) q d x
\end{aligned}
$$

Then, for demonstrate the existence and uniqueness of solution of the problem (1), we announce the following theorem:

Theorem 1 If the functional $\mathrm{F}$ admits a fixed point, then the problem (2) admits a unique solution.

Proof see [4]

And we have:

Theorem 2 The problem (2) admits a unique solution.

Proof. First, if we fixe $w$ and $\theta$ for the first time, and we noted it $w^{1}$ and $\theta^{1}$, we can deduce that the problem (2) admits a unique solution $u^{1}$ (see [3]).

After that, We seek $w^{2}$ from the solution $u^{1}$ in the equation $\varepsilon(u) w=\theta w$, and we apply the same approach, and we found $u^{2}$.

We use this process until we fall to the same solution $w$ which defines the fixed point of $F$.

Then we have:

$$
\begin{aligned}
F\left(w^{2}\right)-F\left(w^{1}\right)= & \int_{\Omega}\left[\left(\beta\left(\operatorname{div}\left(u^{2}\right)\right)^{2}+2 \mu\left(\varepsilon\left(u^{2}\right)\right)^{2}\right] d x\right. \\
& -\int_{\Omega} f \cdot u^{2} d x+\int_{K}\left(\varepsilon\left(u^{2}\right) \cdot w^{2}\right) \lambda^{2} d x \\
& +\int_{K}\left(\varepsilon\left(u^{2}\right) \cdot w^{2}\right) q^{2} d x-\int_{K}\left(\theta^{2} \cdot w^{2}\right) q^{2} d x \\
& -\int_{\Omega}\left[\left(\beta\left(\operatorname{div}\left(u^{1}\right)\right)^{2}+2 \mu\left(\varepsilon\left(u^{1}\right)\right)^{2}\right] d x\right. \\
& +\int_{\Omega} f \cdot u^{1} d x-\int_{K}\left(\varepsilon\left(u^{1}\right) \cdot w^{1}\right) \lambda^{1} d x \\
& -\int_{K}\left(\varepsilon\left(u^{1}\right) \cdot w^{1}\right) q^{1} d x+\int_{K}\left(\theta^{1} \cdot w^{1}\right) q^{1} d x
\end{aligned}
$$




$$
\begin{aligned}
F\left(w^{2}\right)-F\left(w^{1}\right)= & L\left(u^{2}-u^{1}, \lambda^{2}-\lambda^{1}\right) \\
& +\int_{K} \varepsilon\left(u^{2}-u^{1}\right)\left(w^{2}-w^{1}\right)\left(\lambda^{2}-q^{2}\right) d x \\
& +\int_{K}\left(\theta^{2} w^{2}-\theta^{1} w^{1}\right) q^{2} d x
\end{aligned}
$$

so

$$
\begin{aligned}
F\left(w^{2}\right)-F\left(w^{1}\right)= & L\left(u^{2}-u^{1}, \lambda^{2}-\lambda^{1}\right) \\
& +\int_{K}\left(w^{2}-w^{1}\right) \varepsilon\left(u^{2}-u^{1}\right)\left(\lambda^{2}-q^{2}\right) d x \\
& +\int_{K}\left(\varepsilon\left(u^{2}\right) w^{2} \varepsilon\left(u^{1}\right) w^{1}\right) q^{2} d x
\end{aligned}
$$

then

$$
\begin{aligned}
F\left(w^{2}\right)-F\left(w^{1}\right)= & L\left(u^{2}-u^{1}, \lambda^{2}-\lambda^{1}\right) \\
& +\int_{K}\left(w^{2}-w^{1}\right) \varepsilon\left(u^{2}-u^{1}\right)\left(\lambda^{2}-q^{2}\right) d x \\
& +\int_{K}\left(\varepsilon\left(u^{2}\right)\left(w^{2}-w^{1}\right) q^{2}-\varepsilon\left(u^{1}-u^{2}\right) w^{1} q^{2} d x\right.
\end{aligned}
$$

we have two cases,

first case: if $\varepsilon\left(u^{1}-u^{2}\right) w^{1} q^{2}>0$

then, we have

$$
\begin{aligned}
F\left(w^{2}\right)-F\left(w^{1}\right) \leq & L\left(u^{2}-u^{1}, \lambda^{2}-\lambda^{1}\right) \\
& +\int_{K}\left(w^{2}-w^{1}\right) \varepsilon\left(u^{2}-u^{1}\right)\left(\lambda^{2}-q^{2}\right) d x \\
& +\int_{K}\left(\varepsilon\left(u^{2}\right)\left(w^{2}-w^{1}\right) q^{2} d x\right.
\end{aligned}
$$

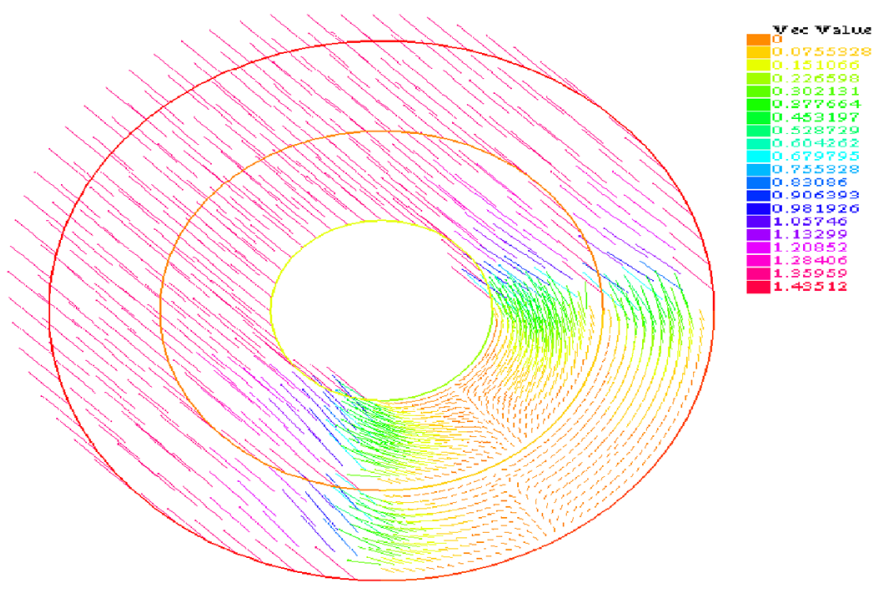

Fig. 1. Case without constraint.

Then

$$
\begin{aligned}
\mid F\left(w^{2}\right)- & F\left(w^{1}\right) \mid \leq\left\|w^{2}-w^{1}\right\|_{L^{2}(K)} \\
& \times\left(\left\|\varepsilon\left(u^{2}-u^{1}\right)\right\|_{L^{2}(K)} \| \lambda^{2}-q^{2}\right) \|_{L^{2}(K)} \\
& \left.+2\left\|\varepsilon\left(u^{2}\right)\right\|_{L^{2}(K)}\left\|q^{2}\right\|_{L^{2}(K)}\right)
\end{aligned}
$$

We deduce that $\mathrm{F}$ is a Lipchitz function, then $\mathrm{F}$ admit a fixed point [12]. Based on Theorem 1, we deduce that the problem (2) has a unique solution.

And since we have an equivalence between the two problems (2) and (1), therefore the problem (1) also has a unique solution.

\section{Numerical results}

and we know that $L\left(u^{2}-u^{1}, \lambda^{2}-\lambda^{1}\right)=0$ because $u^{1}$ and $u^{2}$ are solutions of the initial problem. so

$$
\begin{aligned}
\mid F\left(w^{2}\right)- & F\left(w^{1}\right) \mid \leq\left\|w^{2}-w^{1}\right\|_{L^{2}(K)} \\
& \times\left(\left\|\varepsilon\left(u^{2}-u^{1}\right)\right\|_{L^{2}(K)} \| \lambda^{2}-q^{2}\right) \|_{L^{2}(K)} \\
& \left.+\left\|\varepsilon\left(u^{2}\right)\right\|_{L^{2}(K)}\left\|q^{2}\right\|_{L^{2}(K)}\right) .
\end{aligned}
$$

We deduce that $\mathrm{F}$ is a Lipchitz function, and then $\mathrm{F}$ admits a fixed point.

$$
\text { second case: if } \varepsilon\left(u^{1}-u^{2}\right) w^{1} q^{2} \leq 0
$$

we have

$$
\begin{aligned}
F\left(w^{2}\right)-F\left(w^{1}\right) & =L\left(u^{2}-u^{1}, \lambda^{2}-\lambda^{1}\right) \\
& +\int_{K}\left(w^{2}-w^{1}\right) \varepsilon\left(u^{2}-u^{1}\right)\left(\lambda^{2}-q^{2}\right) d x \\
& +\int_{K}\left(\varepsilon\left(u^{2}\right)\left(w^{2}-w^{1}\right) q^{2}-\varepsilon\left(u^{1}-u^{2}\right) w^{1} q^{2} d x\right.
\end{aligned}
$$

Now we proceed to the processing of this image, and defining the contours by a crown, and we consider the conditions at the edge of type direchlet.

The simulations are performed for the case without stress and the case with strain constraint for the elasticity problem, and we obtain the following results:

\subsection{Comment}

In Figure 1, we have presented the case where we have no constraints. Then, in Figure 2, and based on the result of Figure 1, it can be clearly seen that the displacement follows the direction of the core fibers, even if one changes direction as in Figure 3, always the displacement follows the direction of the fibers of the heart.

On the other hand, if the direction of the fibers of the heart is fixed, at the same time the displacement is initiated by a direction against the fixed one of the displacement, one falls on a result of an almost null displacement, which is the case in Figure 4.

In the Figure 5, we can deduce that the deformation so

$$
\begin{aligned}
F\left(w^{2}\right)- & F\left(w^{1}\right) \leq L\left(u^{2}-u^{1}, \lambda^{2}-\lambda^{1}\right) \\
& +\int_{K}\left(w^{2}-w^{1}\right) \varepsilon\left(u^{2}-u^{1}\right)\left(\lambda^{2}-q^{2}\right) d x \\
& +\int_{K} 2 \varepsilon\left(u^{2}\right)\left(w^{2}-w^{1}\right) q^{2} d x
\end{aligned}
$$
follows the direction in the case of eigenvalues and eigenvectors, and it's the general and real case.

If we summarize, we can say that it has been shown that the depletion $\mathrm{u}$ of a point of the wall of the left ventricle follows the direction of the orientation of the fibers of the left ventricle in the deformation. 


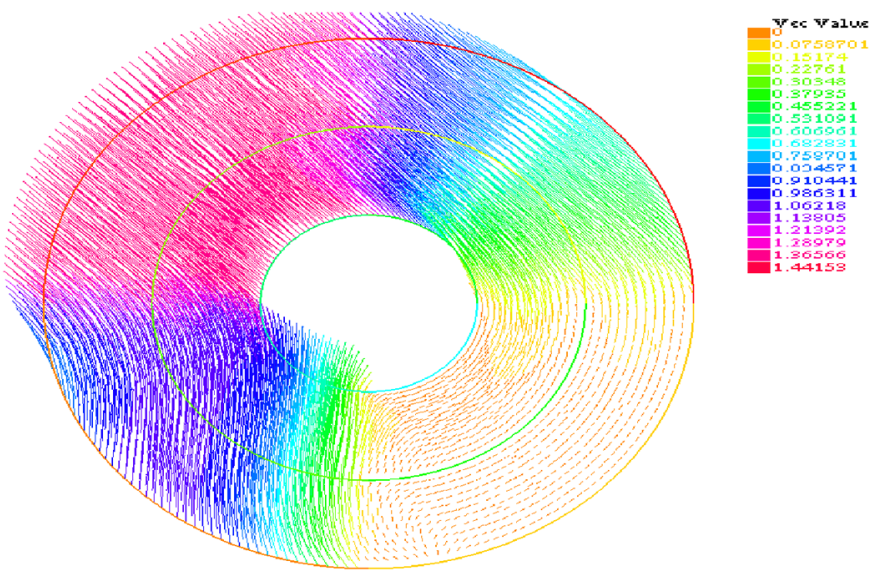

Fig. 2. First case with constraint of displacement $\mathrm{u}$ in direction $w$.

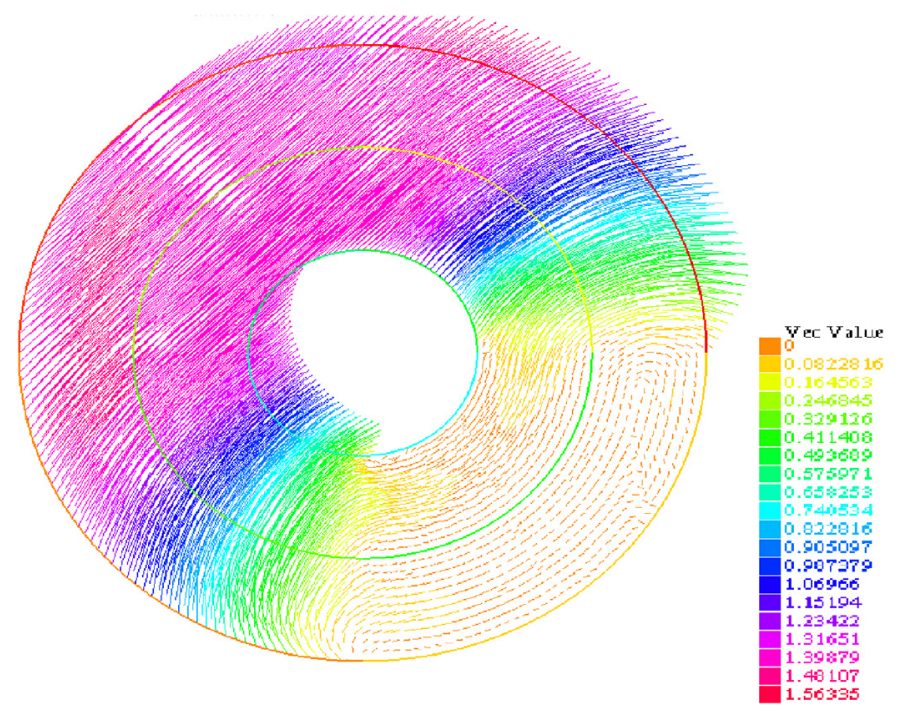

Fig. 3. Second case with constraint of displacement $u$ in direction $w$.

\section{Image processing of the heart}

In this section, we will focus on the processing of an image of the heart, especially the left ventricle, for the application of the simulations made above [13-15]. In this case, we take the following image, mentioned in Figure 6, of the left ventricle taken by an MRI.

We define the contours of the left ventricle in the previous image. In this case, we want to define the most corrected contours to make a good segmentation of the left ventricle. Based on the previous results, we control the direction of the deformation to calculate the thickness of the walls and therefore define the radius of the contours of the segmentation, and we have the results in Figure 7, as follow:

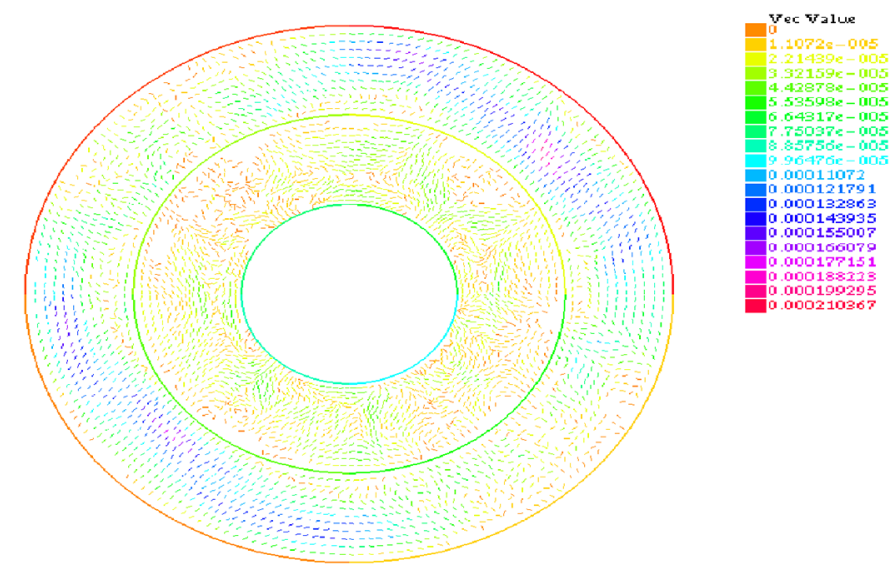

Fig. 4. Case with constraint where the displacement $\mathrm{u}$ does not follow the direction $w$.

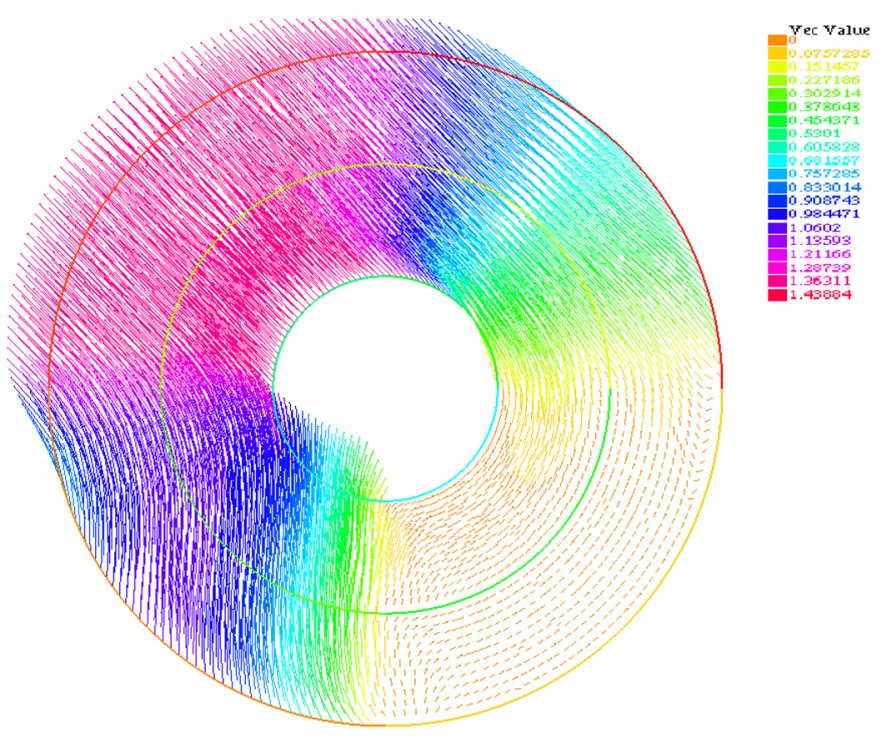

Fig. 5. Case of eigenvalues and eigenvectors.

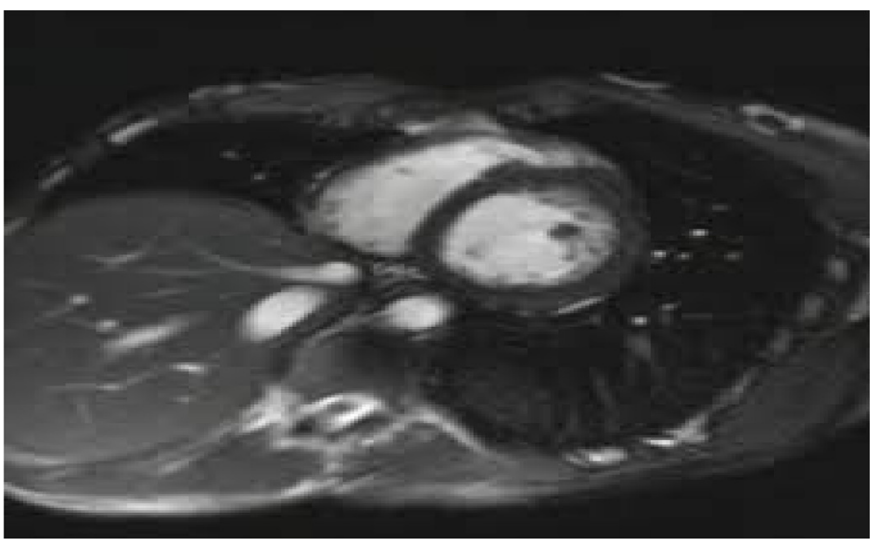

Fig. 6. Heart image taken by an MRI. 


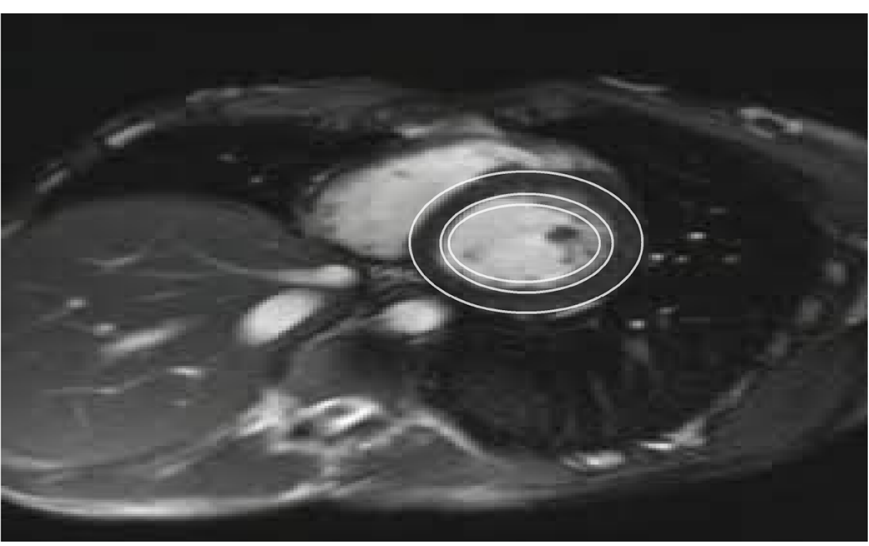

Fig. 7. Segmentation of an image of the heart taken by an MRI.

\section{Conclusion}

As a conclusion, this work has been devoted to study the problem of elasticity under pressure to consider the direction of displacement of the structure.

We have seen, first, the existence and uniqueness of solution of the elasticity model with the stress of deformation.

Then we performed numerical simulations in FreeFem for our proposed model of elasticity, with different cases of direction of displacement, with and without stress.

The results of this study are useful for a good extraction of the characteristics of the information in a cardiac image, in particular, as regards detection of the contours of the left ventricle of the heart, under stress of deformation.

We are thankful to the Financial support from the entrepreneurship and development laboratory, Faculty of Legal, Economic and Social Sciences of SALE, Mohammed V University. This allow us to get the best working conditions within our scientific research community in Morocco.

\section{References}

1. V. Manet, Méthode des Éléments Finis: vulgarisation des aspects mathématiques et illustration de la méthode, ViM2, Lyon, 2018, pp. 306

2. A. Khalil, S.-C. Ng, Y.M. Liew, K.W. Lai, An overview on image registration techniques for cardiac diagnosis and treatment, Hindawi J. 2018, 1437125 (2018)

3. A. Azzayani, S. Boujena, J. Pousin, A model of elasticity taking into account the displacement orientation in the deformation, Br. J. Math. Comput. Sci. (2016)

4. A. Azzayani, S. Boujena, J. Pousin, Sur un modèle d'élasticité non isotrope tenant compte de l'orientation du déplacement pour la segemntation d'images, Troisième Congrés de la SM2A, Marrakech-Maroc, 10-13 Septembre 2012

5. F. Boyer, Analyse numérique des EDP elliptiques, Master 2ième année - Mathématiques et Applications, Aix-Marseille universités, 2016

6. A. Azzayani, S. Boujena, J. Pousin, Un modèle d'élasticité 2D tenant compte de l'orientation du déplacement, TAMTAM - Alger, 2013

7. A. Azzayani, S. Boujena, J. Pousin, A 3D model of elasticity taking into account the orientation of the displacement in the deformation, J. d'Analyse Numér. d'Optim. (2013)

8. A. Ern, J.-L. Guermond, Theory and Practice of Finite Elements (Springer, 2018)

9. B. Susanne, S. Ridgway, The Mathematical Theory of Finite Element Methods (Springer, 2018)

10. P.A. Raviart, J.M. Thomas, Introduction à l'analyse numérique des équations aux dérivées partielles (Dunod editions, 2016)

11. G. Allaire, Analyse numérique et optimisation (Editions Ecole Polytechnique, 2017)

12. Frederic Hecht, FreeFem ++ v 4.6, May 202020

13. J-P. Demailly, Analyse numérique et équations différentiel, Les collections Grenoble Sciences, presses universitaires de Grenoble, Grenoble, 2006

14. M.R. Avendi, A. Kheradvar, H. Jafarkhani, A combined deep-learning and deformable-model approach to fully automatic segmentation of the left ventricle in cardiac MRI, Med. Image Anal. 30, 108-119 (2016)

15. F. Luca, Q. Alfio, V. Alessandro, Cardiovascular Mathematics: Modeling and simulation of the circulatory system (Springer editions, 2019), 2006 - 343 pages 